\title{
Le divorce en droit international privé
}

Silvia Pfeiff

Avocate

Assistante à l'ULB et à l'Ulg 


\section{La compétence internationale}

$\square$ Quel texte?

- Le Règlement 2201/2003 du 27 novembre 2003, relatif à la compétence, la reconnaissance et l'exécution des décisions en matière matrimoniale et en matière de responsabilité parentale (Règlement Bruxelles IIbis)

- Le Code de droit international privé 


\section{A. Le Règlement Bruxelles IIbis}

Entrée en vigueur le $1^{\text {er }}$ mars 2005

Concerne la dissolution du lien matrimonial (et la responsabilité parentale)

Principe de l'exclusivité des règles européennes à l'égard des défendeurs dit « communautaires » (article 6)

$\square$ Application subsidiaire du Code lorsque aucune juridiction d'un Etat membre ne peut asseoir sa compétence sur base du Règlement Bruxelles II bis (article 7) 


\section{B. Chefs de compétence (article 3 du Règlement)}

\section{$\square$ Critère de la résidence habituelle}

- La résidence habituelle actuelle des époux

- La dernière résidence habituelle des époux ssi l'un des époux y réside encore

- La résidence habituelle du défendeur

- La résidence habituelle de l'un des époux en cas de demande conjointe

- La résidence habituelle du demandeur : un an, sauf si ressortissant de cet Etat (6 mois)

\section{$\square$ Critère de la nationalité}

- Etat dont les deux époux sont ressortissants 


\section{Si je ne parviens pas à justifier la compétence des juridictions belges}

口 Vérifier si un autre Etat membre est compétent sur base du Règlement

(Art. 17; CJ Sundelind Lopez 29 nov. 2007)

\section{$\square$ A défaut, puis-je appliquer le Codip?}

- Art. 7 : Lorsque aucune juridiction d'un État membre n'est compétente en vertu des articles 3, 4 et 5, la compétence est, dans chaque État membre, réglée par la loi de cet État.

- Art. 6 :Un époux qui:

a) a sa résidence habituelle sur le territoire d'un État membre, ou

b) est ressortissant d'un État membre ou, dans le cas du Royaume-Uni et de l'Irlande, a son "domicile" sur le territoire de l'un de ces États membres,

ne peut être attrait devant les juridictions d'un autre État membre qu'en vertu des articles 3, 4 et 5 . 


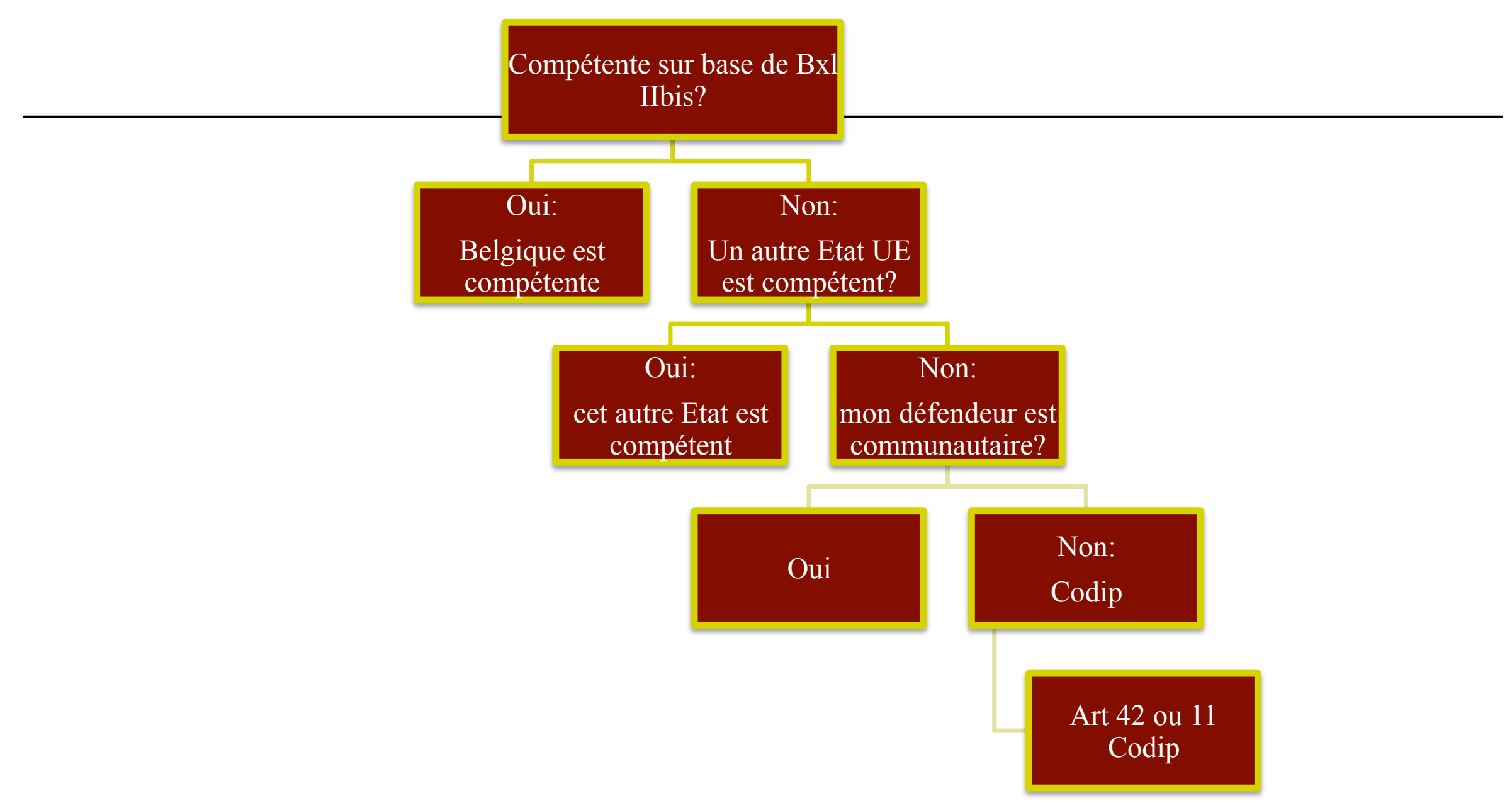




\section{Le code de droit international privé}

․ Art. 42 du Codip

$1^{\circ}$ En cas de demande conjointe, résidence habituelle de l'un des époux en Belgique

$2^{\circ}$ la dernière résidence habituelle commune des époux se situait en Belgique moins de douze mois avant l'introduction de la demande;

$3^{\circ}$ l'époux demandeur a sa résidence habituelle depuis douze mois au moins en Belgique lors de l'introduction de la demande; ou $4^{\circ}$ les époux sont belges lors de l'introduction de la demande.

Art. 11 du Codip

$\square$ Art. 5 du Codip 


\section{La loi applicable}

$\checkmark$ Pas de réglementation européenne

-> Application du Code de droit international privé

(art. 55 du Code) 


\section{Rattachement en cascade}

1. Le droit de l'Etat sur le territoire duquel l'un et l'autre époux ont leur résidence habituelle lors de l'introduction de la demande

2. A défaut, la loi de la dernière résidence habituelle commune des époux ssi l'un des époux a conservé sa résidence dans cet Etat au moment de l'introduction de la demande

3. A défaut, la loi de la nationalité commune des époux

4. A défaut, le droit belge 


\section{Clause de sauvegarde}

Art. 55 § 3 du Code:

"L'application du droit désigné au § ler est écartée dans la mesure où ce droit ignore l'institution du divorce. Dans ce cas, il est fait application du droit désigné en fonction du critère établi de manière subsidiaire par le ler» 


\section{Choix de la loi applicable par les époux}

$\square$ Art. 55 § 2 du Code:

Choix limité:

- soit le droit belge

- soit le droit de leur nationalité commune

Le choix doit être exprimé lors de la première comparution 


\section{La reconnaissance des}

décisions de dissolution du mariage 


\section{A. Au sein de l'UE (à l'exception du Danemark)}

$\square$ Règlement Bruxelles II bis

$\checkmark$ Principe: reconnaissance automatique des décisions d'annulation du mariage, de séparation de corps et de divorce

$\checkmark$ Pas de reconnaissance automatique d'une décision de rejet 


\section{Motifs de non reconnaissance}

(art. 22Règlement Bruxelles II bis)

$\square$ Violation de l'ordre public de l'Etat requis

$\square$ Violation des droits de la défense au sens strict (absence de signification de l'acte introductif à moins que le défendeur n'ai accepté la décision)

$\square$ Décision inconciliable avec une décision rendue dans l'Etat membre ou antérieurement dans un autre Etat et susceptible d'être reconnue 


\section{Reconnaissance d'une décision rendue par un}

\section{Etat membre}

$\square$ Documents à produire (art. 37 du Règlement)

- l'expédition de la décision

- le certificat visé par l'article 39

- si décision par défaut, preuve que l'acte introductif a été notifié ou signifié ou que le défendeur a accepté la décision 


\section{Les décisions émanant d'un Etat hors UE ou du Danemark}

$\square$ Principe: reconnaissance automatique (art. 22 Codip)

․ Motifs de refus (art. 25 Codip)

- Violation de l'ordre public

- Violation des droits de la défense

- Fraude à la loi

- Inconciliable avec décision antérieure

- Demande pendante en Belgique entre mêmes parties et ayant même objet

- Juridictions Belges étaient seules compétentes

- Compétence uniquement fondée sur présence du défendeur ou de biens

- Reconnaissance se heurte à un motif de refus particulier (not. Art 57) 
Reconnaissance d'une décision non communautaire

\section{Il faut produire:}

- l'expédition de la décision + légalisation (sauf dispense)

- La preuve que la décision est exécutoire et qu'elle a été signifiée ou notifiée

- Si décision par défaut, preuve que l'acte introductif d'instance a été notifié ou signifié 
Cas particulier des répudiations 


\section{Aperçu de droit marocain}

$\square$ Différents types de dissolution du mariage depuis l'entrée en vigueur du nouveau Code marocain de la famille

$\square \quad$ La répudiation Talak

La répudiation moyennant compensation (Kohl)

La répudiation Tamlik

$\square \quad$ Le divorce pour discorde (Chiqaq)

$\square$ Divorce qui peut être sollicité uniquement par épouse

- Défaut d'entretient, vice rédhibitoire, absence 


\section{Principe: Art. 57 Codip}

$\S 1$ er. Un acte établi à l'étranger constatant la volonté du mari de dissoudre le mariage sans que la femme ait disposé d'un droit égal ne peut être reconnu en Belgique.

$\square \quad \S 2$. Toutefois, un tel acte peut être reconnu en Belgique après vérification des conditions cumulatives suivantes :

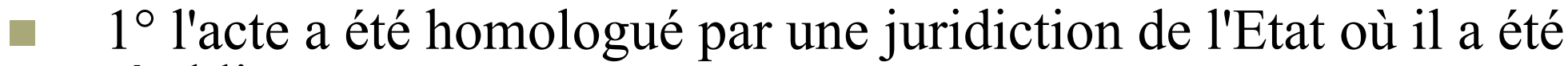
établi;

- $\quad 2^{\circ}$ lors de l'homologation, aucun époux n'avait la nationalité d'un Etat dont le droit ne connaît pas cette forme de dissolution du mariage;

- $\quad 3^{\circ}$ lors de l'homologation, aucun époux n'avait de résidence habituelle dans un Etat dont le droit ne connaît pas cette forme de dissolution du mariage;

- $\quad 4^{\circ}$ la femme a accepté de manière certaine et sans contrainte la dissolution du mariage;

- $\quad 5^{\circ}$ aucun motif de refus visé à l'article 25 ne s'oppose à la reconnaissance. 